Research Article

\title{
Rotor Blades Diagnosis Method Based on Differences in Phase Shifts
}

\author{
Rafał Grądzki (D), ${ }^{1}$ Paweł Lindstedt, ${ }^{2}$ Zbigniew Kulesza ${ }^{(D},{ }^{1}$ and Błażej Bartoszewicz ${ }^{1}$ \\ ${ }^{1}$ Faculty of Mechanical Engineering, Bialystok University of Technology, Wiejska 45C, 15-351 Bialystok, Poland \\ ${ }^{2}$ Air Force Institute of Technology, Ksiecia Boleslawa 6A, 01-494 Warsaw, Poland \\ Correspondence should be addressed to Zbigniew Kulesza; z.kulesza@pb.edu.pl
}

Received 17 June 2018; Revised 13 September 2018; Accepted 20 September 2018; Published 21 October 2018

Academic Editor: Paolo Pennacchi

Copyright (C) 2018 Rafał Grądzki et al. This is an open access article distributed under the Creative Commons Attribution License, which permits unrestricted use, distribution, and reproduction in any medium, provided the original work is properly cited.

In this work, an innovative diagnosis method of rotor blades is presented. A measurement signal of blade tip displacement is divided into two observation zones, in which two peaks of an additional template signal are introduced. Cross-correlation and power spectral density functions of the measurement and template signals in these two distinct zones are calculated. Next, the phase shift between the two cross-correlation functions is obtained by calculating the ratio of the two power spectral density functions. The changes in parameters of an analytical description of this phase shift are used to determine a distinct and easy-toanalyze image of the technical condition of the tested blade. A graphical portrait indicating the technical condition of all blades in the annulus is developed. Although not directly measured, environmental signals (e.g., external disturbances and sensor's noise) affecting the diagnostic process are included in the method. It is demonstrated that, by using the proposed signal processing technique, a negative influence of these disturbances is eliminated and the reliability of the machine technical condition indications is improved. The approach is demonstrated using experimental results of turbine engine blade displacements. High effectiveness of the method and measurement equipment has been confirmed in a wide range of analyzed tip-timing results obtained in different periods of the measurement process.

\section{Introduction}

One of the basic components of rotating machines on which reliable and safe operation depends is rotor blades. Practice proves that breaking off even a single blade out of several tens or even hundreds in a bladed rotor almost always leads to a serious machine failure and consequently to a tragic catastrophe. The knowledge about the technical state of the blade is also important to avoid unplanned machine downtimes and to plan the overhauls accurately. Therefore, the technical condition of the machine, and especially of its rotor blades, should be monitored continuously to detect damages as quickly as possible.

Several methods of rotor blades diagnosis have been developed and tested, such as eddy current [1], ultrasonic $[2,3]$, thermographic $[4,5]$, radiographic $[4,6]$, and vibration methods [7-10]. A comprehensive literature review of various blade faults detection methods has been presented by Abdelrhman et al. [11].

The most popular approach that can be applied for rotating blades is the frequency domain analysis of blade vibrations. The wide vibration spectrum of the tested machine can be compared with a vibration spectrum library to detect various blade faults such as foreign object damage, blade loss part, blade rub, or loose joints [7]. Different changes in blade pass frequency and its harmonics are reported as early indications of the occurring blade-casing rubbing in steady rotation $[8,9]$ or in the runup or coast-down [10] of the machine. A modification of this approach based on cepstrum analysis has been applied to various blade faults by Satyam et al. [12] and Randall and Sawalhi [13]. Chang and Chen [14] used wavelet analysis methods to detect cracked blades. A similar approach has been applied by Aretakis and Mathioudakis [15], who demonstrated that each type of the analyzed blade faults 
(fouled, twisted, and mistuned blade) generates a unique wavelet signature. Lim and Leong [16] tested wavelet analysis methods for loose, rubbing, or creeping blades. Possible applications of ensemble empirical mode decomposition (EEMD), multiwavelet packet transform, Hilbert-Huang transform for multifault identification in a bladed rotor have been studied by Jiang et al. [17].

Another variant of the blade vibration analysis, the socalled tip-timing method [18], involves the extraction of the vibrations of a single blade from a measurement signal obtained from displacement sensors. This signal contains the displacements of other blades. The extracted vibrations of this single blade can then be analyzed for possible occurrence of blade rubbing [19], blade cracks or loosing [20], or other blade faults $[21,22]$ by analyzing time differences of blade passages in different rotor revolutions. The tip-timing method requires special measurement equipment [23-26] and has its long history, well presented by Rzadkowski et al. [27].

Displacement, velocity, and acceleration of blade tips are not the only quantities that can be used as diagnostic signals in the vibration-based diagnostic methods. Temperature $[28,29]$, pressure, and flow fluctuations $[15,30]$ inside machine chambers have also been used to detect various blade faults. A popular approach includes measurement of different parameters of acoustic emission [15, 31, 32] to detect blade faults.

All these signals can be termed as "operational signals" because they are directly measured, and they carry on the information about the operational parameters of the machine, i.e., about its technical condition. However, there are many other signals such as measurement noises or external electromagnetic disturbances that affect the normal operation of the machine and of the measurement process but are difficult or even impossible to be measured directly. All these disturbing signals can be termed as "environmental signals," as they carry on the information about the parameters of the technical and natural environment surrounding the machine. Usually, environmental signals are not included in traditional blade diagnostic methods, yet their influence on the obtained fault indications may be significant.

The proposed method is based on a diagnostic model of the blade and includes the phase shift between crosscorrelation functions of the displacement signal and an additional template signal calculated in two observation zones: the first zone when the blade tip approaches the sensor and the other zone when the blade tip recedes the sensor. In this model, environmental signals are included and it is demonstrated that, by using the proposed signal processing technique, a negative influence of these disturbances is eliminated. This way the reliability of the machine technical condition indications is improved. The method has been developed by Lindstedt and Gradzki [33-36], and the current paper improves and extends the approach by consolidating the form of the diagnostic model and the forms of damage maps. Furthermore, it introduces a new, compact form of a graphical damage representation to quickly assess the technical condition of all blades in the annulus. Provided experimental results confirm high effectiveness of the method in a wide range of analyzed blade tip vibrations obtained in different periods of the measurement process. An important part of the measurement equipment is a specially designed contactless inductive sensor, which is essential for a correct application of the method.

It should be emphasized that the proposed method can be used to assess the technical condition of a blade in its normal operation. The analysis is conducted online. All external forces affecting this operation are included. No disassembly of an engine to perform static tests of the blades is required (which currently is a common practice). The method can be used to detect different types of blade malfunctions (e.g., mechanical wear or damage, fatigue crack, blade nicking or bending, and high-temperature damage) and gives early damage indications to plan overhauls accurately.

\section{Diagnostic Model}

2.1. Mathematical Description. Usually only one signal of blade tip displacement $y(t)$ (apart from the rotating speed $\Omega$ ) is directly measured. This signal is further termed as the "operational signal." Operational signal is often disrupted with some sensor noise or other disturbances. The influence of these disturbing factors can be included by introducing an additional environmental signal $x(t)$. This abstract signal $x(t)$, gathering all possible environmental disturbances, is present in the operational signal $y(t)$, but it is difficult or even impossible to be measured. However, its influence on the diagnostic procedure results may be significant. Usually, environmental signal is represented as white noise.

A typical operational signal of blade tip displacements $y(t)$ obtained using the tip-timing procedure is presented in Figure 1 . The peaks in $y(t)$ represent the so-termed cycles of blade tip operation.

Using the proposed method, the signal $y(t)$ is divided into two observation zones at each cycle. The first zone of time length $\Delta t_{1}$ is from $t_{0}$ to $t_{1}$ and it is termed as the approaching zone. At this zone, the blade tip approaches the displacement sensor. The other zone is from $t_{1}$ to $t_{2}$, its time length is $\Delta t_{2}$, and it is termed as the receding zone. At this zone, the blade tip recedes the sensor. The time $t_{1}$ is related to the moment when the blade tip is just beneath the displacement sensor.

Next, additional template signal $z(t)$ is introduced. This signal is assumed as a series of approximations $\widehat{\delta}(t)$ of Dirac's delta distribution $\delta(t)$, where

$$
\begin{array}{r}
\delta(t)= \begin{cases}0, & t \neq t_{m}, \\
+\infty, & t=t_{m},\end{cases} \\
\int_{-\infty}^{+\infty} \delta(t) d t=1 .
\end{array}
$$

The peaks of these approximations $\widehat{\delta}(t)$ are located in the middle $t_{m}=\left(t_{1}-\Delta t_{1}\right)$ of the approaching zone and in the middle $t_{m}=\left(t_{1}+\Delta t_{2}\right)$ of the receding zone of the operational signal $y(t)$ (Figure 1 ). As $\widehat{\delta}(t)$, any approximation of Dirac's distribution $\delta(t)$ may be used [37]. The only 


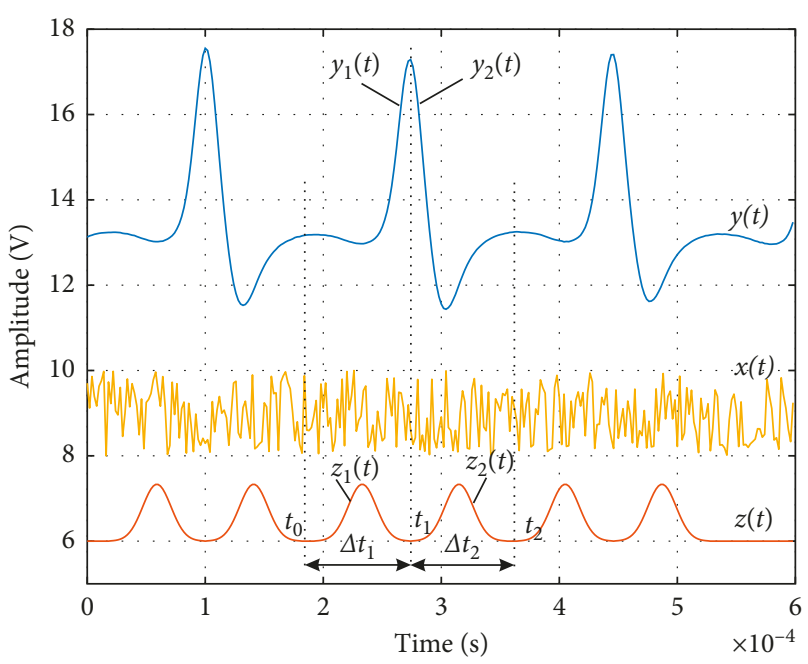

FIgURE 1: Typical blade tip displacement (operational) signal $y(t)$, environmental signal $x(t)$, and the proposed template signal $z(t)$.

requirement is that there must be some correlation between $\widehat{\delta}(t)$ and $y(t)$.

The cross-correlation functions $R_{z y 1}(\tau)$ and $R_{z y 2}(\tau)$ between operational $y(t)$ and template signal $z(t)$ are then calculated in separate zones $\Delta t_{1}$ and $\Delta t_{2}$ as

$$
\begin{aligned}
& R_{z y 1}(\tau)=\int_{t_{0}}^{t_{1}} z(t) y(t-\tau) d t, \\
& R_{z y 2}(\tau)=\int_{t_{1}}^{t_{2}} z(t) y(t-\tau) d t,
\end{aligned}
$$

where $\tau$ is the time shift variable.

Next, using the Wiener-Khinchin theorem, power spectral density functions $S_{z y 1}(\omega)$ and $S_{z y 2}(\omega)$ are calculated as Fourier transforms of the corresponding cross-correlation functions $R_{z y 1}(\tau)$ and $R_{z y 2}(\tau)$ [38]:

$$
\begin{aligned}
& S_{z y 1}(\omega)=\mathbb{F}\left[R_{z y 1}(\tau)\right], \\
& S_{z y 2}(\omega)=\mathbb{F}\left[R_{z y 2}(\tau)\right],
\end{aligned}
$$

where $\mathbb{F}[f(\tau)]$ denotes the Fourier transform of a time function $f(\tau)$.

Using the corresponding form of Equation (3), the power spectral density functions $S_{z x 1}(\omega)$ and $S_{z x 2}(\omega)$ are calculated, where the autocorrelation functions $R_{z x 1}(\tau)$ and $R_{z x 2}(\tau)$ between the template $z(t)$ and environmental $x(t)$ signals in the two separate observation zones are defined as

$$
\begin{aligned}
& R_{z x 1}(\tau)=\int_{t_{0}}^{t_{1}} z(t) x(t-\tau) d t, \\
& R_{z x 2}(\tau)=\int_{t_{1}}^{t_{2}} z(t) x(t-\tau) d t .
\end{aligned}
$$

The obtained power spectral density functions $S_{z y 1}(\omega)$, $S_{z y 2}(\omega), S_{z x 1}(\omega)$, and $S_{z x 2}(\omega)$ are then used to present the relation between operational $y(t)$ and environmental $x(t)$ signals as

$$
\begin{aligned}
& \varphi_{1}(\omega)=\operatorname{Arg}\left(\frac{S_{z y 1}(\omega)}{S_{z x 1}(\omega)}\right), \\
& \varphi_{2}(\omega)=\operatorname{Arg}\left(\frac{S_{z y 2}(\omega)}{S_{z x 2}(\omega)}\right),
\end{aligned}
$$

where $\varphi_{1}(\omega)$ is a phase shift between signals $R_{z y 1}(\tau)$ and $R_{z x 1}(\tau)$ at the approaching zone, $\varphi_{2}(\omega)$ is a phase shift between signals $R_{z y 2}(\tau)$ and $R_{z x 2}(\tau)$ at the receding zone, and $\operatorname{Arg}(G(\omega))$ denotes the function which calculates the argument (the angle) of some complex function $G(\omega)$.

If time intervals $\Delta t_{1}$ and $\Delta t_{2}$ are close to each other (i.e., when $\Delta t_{2}$ immediately follows $\Delta t_{1}$ ), then power spectral density functions $S_{z x 1}(\omega)$ and $S_{z x 2}(\omega)$ can be considered as equal, i.e.,

$$
S_{z x 1}(\omega)=S_{z x 2}(\omega)=S_{z x}(\omega) .
$$

This assumption can be explained by the observation that if the time distance between intervals $\Delta t_{1}$ and $\Delta t_{2}$ is short, then environmental signal $x(t)$ in those time intervals may be considered as unchanged (or only slightly changed in a small degree).

Using Equations (5) and (6), the difference between phase shifts $\varphi_{2}(\omega)$ and $\varphi_{1}(\omega)$ can be calculated as

$$
\begin{aligned}
\varphi_{21}(\omega) & =\varphi_{2}(\omega)-\varphi_{1}(\omega)=\operatorname{Arg}\left(\frac{S_{z y 2}(\omega) / S_{z x 2}(\omega)}{S_{z y 1}(\omega) / S_{z x 1}(\omega)}\right) \\
& =\operatorname{Arg}\left(\frac{S_{z y 2}(\omega)}{S_{z x}(\omega)} \times \frac{S_{z x}(\omega)}{S_{z y 1}(\omega)}\right)=\operatorname{Arg}(G(\omega)),
\end{aligned}
$$

where

$$
G(\omega)=\frac{S_{z y 2}(\omega)}{S_{z y 1}(\omega)}
$$

Note that according to Equation (3), the ratio of power spectral densities $S_{z y 2}(\omega)$ and $S_{z y 1}(\omega)$ is a transfer function denoted as $G(\omega)$. The argument of this function is the phase shift between signal $R_{z y 2}(\tau)$ at the receding zone and signal $R_{z y 1}(\tau)$ at the approaching zone. This argument $\varphi_{21}(\omega)$ can be considered as a unified representation of the technical condition of the tested blade, i.e., as a diagnostic model of the blade.

Also note that by including Equation (6), the power spectral density functions $S_{z x 1}(\omega)$ and $S_{z x 2}(\omega)$ are eliminated from the model. That means that direct measurements of the environmental signal $x(t)$ are not required. Of course, indirectly, this signal is included in the model, as it is a part of the operational signal. Thanks to a special organization of the diagnostic procedure, in which the analysis is conducted in two separate observation zones, and the ratio of two power spectral density functions $S_{z y 1}(\omega)$ and $S_{z y 2}(\omega)$ is calculated, and the influence of the environmental signal is eliminated.

2.2. Selection of Template Signal. The template signal $z(t)$ can be chosen as any periodic function of time, i.e., the function which repeats the same pattern in time intervals 
$\Delta t_{1}$ and $\Delta t_{2}$ for subsequent blades. If this template signal repeats the same pattern in subsequent time intervals, then the changes in cross-correlation $R_{z y 1}(\tau)$ and $R_{z y 2}(\tau)$ functions (and consequently in the phase shift $\varphi_{21}(\omega)$ between these cross-correlations) in the receding and approaching zones can appear only due to the changes in signal $y(t)$. Certainly, it is supposed that the changes in the operational signal $y(t)$ reflect the changes in the technical condition of the blade.

This is the main requirement for the template signal: periodically repeated patterns in subsequent time intervals $\Delta t_{1}$ and $\Delta t_{2}$. The other one is there should be some correlation between $z(t)$ and $y(t)$. Specifically, the template signal $z(t)$ can be selected as a series of Dirac's delta distributions (as proposed in the current paper) to better reconstruct the shape of the operational signal $y(t)$ and consequently to obtain more robust values of correlation $R_{z y 1}(\tau), R_{z y 2}(\tau)$ and power density $S_{z y 1}(\omega), S_{z y 2}(\omega)$ functions.

Another reason for introducing the template signal $z(t)$ is to correlate it with the environmental signal $x(t)$ (which is always present in the operational signal $y(t)$ ). If this template signal is identical in subsequent time intervals $\Delta t_{1}$ and $\Delta t_{2}$ and if the time distance between these intervals is small, then the assumption about identical cross-correlation functions $R_{z x 1}(\tau)$ and $R_{z x 2}(\tau)$ (and consequently about power spectral density $S_{z x 1}(\omega), S_{z x 2}(\omega)$ functions) can be taken. Finally, the influence of the environmental signal $x(t)$ can be eliminated (as explained by Equation (7)). Of course, only the constant environment gives right to take such assumption, i.e., the environment in which the values of environmental signal $x(t)$ do not change considerably between time intervals $\Delta t_{1}$ and $\Delta t_{2}$.

\section{Blade Condition Monitoring Using the New Diagnostic Model}

To simplify further considerations, it is assumed that $t_{1}=0$ (Figure 1), i.e., the analyzed peak in signal $y(t)$ is shifted to the beginning of the coordinate system. The operational signal $y(t)$ of a given blade is measured and sampled with time period $h>0$. Then, prescriptive numbers $(N+1)$ of signal samples in two adjacent observation zones $\Delta t_{1}$ and $\Delta t_{2}$ are collected into two separable sets $y_{1}\left(n_{1}\right)$ and $y_{2}\left(n_{2}\right)$, where $n_{1}=-N, \ldots,-1,0$ and $n_{2}=0,1, \ldots, N$ (Figure 1). The number $(N+1)$ of signal samples in each set must be chosen carefully to ensure proper statistical assessment of the measured operational signal $y_{1}\left(n_{1}\right)$ and $y_{2}\left(n_{2}\right)$.

Next, to reduce frequency leakage, the signals $y_{1}\left(n_{1}\right)$ and $y_{2}\left(n_{2}\right)$ are scaled by Hanning window $H_{\mathrm{w}}(n)$ :

$$
H_{\mathrm{w}}(n)=\frac{1}{2}\left(1-\cos \left(\frac{2 \pi n}{N}\right)\right),
$$

to form new signals $y_{\mathrm{H} 1}\left(n_{1}\right)$ and $y_{\mathrm{H} 2}\left(n_{2}\right)$ :

$$
\begin{aligned}
& y_{\mathrm{H} 1}\left(n_{1}\right)=H_{\mathrm{w} 1}\left(n_{1}\right) y_{1}\left(n_{1}\right), \\
& y_{\mathrm{H} 2}\left(n_{2}\right)=H_{\mathrm{w} 2}\left(n_{2}\right) y_{2}\left(n_{2}\right) .
\end{aligned}
$$

Discrete approximations $\widehat{\delta}\left(n_{1}\right)$ and $\widehat{\delta}\left(n_{2}\right)$ of Dirac's delta distribution $\delta(t)$ are chosen as

$$
\begin{aligned}
& \widehat{\delta}\left(n_{1}\right)=\frac{1}{T} e^{-\left(\pi\left(2 n_{1}+N\right)^{2} / T^{2}\right),} \\
& \widehat{\delta}\left(n_{2}\right)=\frac{1}{T} e^{-\left(\pi\left(2 n_{2}-N\right)^{2} / T^{2}\right),}
\end{aligned}
$$

to form template signals $z_{1}\left(n_{1}\right)$ and $z_{2}\left(n_{2}\right)$ in the approaching and receding zones, where $z_{1}\left(n_{1}\right)=\widehat{\delta}\left(n_{1}\right)$, $z_{2}\left(n_{2}\right)=\widehat{\delta}\left(n_{2}\right)$, and parameter $T$ changes the amplitude of approximation.

Then, discrete cross-correlation functions $R_{z y 1}\left(n_{2}\right)$ and $R_{z y 2}\left(n_{2}\right)$ between the signals $y_{\mathrm{H} 1}\left(n_{1}\right), y_{\mathrm{H} 2}\left(n_{2}\right)$ and $z_{1}\left(n_{1}\right)$, $z_{2}\left(n_{2}\right)$ are calculated, as follows:

$$
\begin{aligned}
& R_{z y 1}\left(n_{2}\right)=\sum_{l=0}^{N-n_{2}-1} z_{1}\left(l-n_{2}-N+1\right) y_{\mathrm{H} 1}(l-N+1), \\
& R_{z y 2}\left(n_{2}\right)=\sum_{l=0}^{N-n_{2}-1} z_{1}\left(l+n_{2}\right) y_{\mathrm{H} 1}(l) .
\end{aligned}
$$

Cross-correlation functions $R_{z y 1}\left(n_{2}\right)$ and $R_{z y 2}\left(n_{2}\right)$ are then approximated with smooth approximating functions to obtain analytical representations $R_{z y 1}(\tau)$ and $R_{z y 2}(\tau)$. Approximating functions polynomials may be used [35], resulting in the following forms of the cross-correlation functions:

$$
\begin{aligned}
& R_{z y 1}(\tau)=a_{r} \tau^{r}+\mathrm{a}_{r-1} \tau^{(r-1)}+\cdots+a_{1} \tau+a_{0}, \\
& R_{z y 2}(\tau)=b_{r} \tau^{r}+b_{r-1} \tau^{(r-1)}+\cdots+b_{1} \tau+b_{0},
\end{aligned}
$$

where $a_{i}$ and $b_{i}$ are coefficients of the polynomials, $i=0,1,2, \ldots, r$.

The order $r$ of the polynomials should be chosen carefully: too low order will result in inaccurate approximations, too high order will result in an excessive number of polynomial coefficients and longer calculation times.

Based on analytical representations of the crosscorrelation functions $R_{z y 1}(\tau), R_{z y 2}(\tau)$, Laplace transforms $\mathbb{F}\left[R_{z y 1}(\tau)\right], \mathbb{F}\left[R_{z y 2}(\tau)\right]$ and power spectral density functions $S_{z y 1}(\omega), S_{z y 2}(\omega)$ are calculated according to Equation (3). Next, the power spectral density functions $S_{z y 1}(\omega), S_{z y 2}(\omega)$ are introduced into Equations (8) and (7). For polynomial approximation, the diagnostic model $\varphi_{21}(\omega)$ is obtained in the following form:

$$
\begin{aligned}
\varphi_{21}(\omega) & =\operatorname{Arg}\left(\frac{S_{z y 2}(\omega)}{S_{z y 1}(\omega)}\right) \\
& =\operatorname{Arg}\left(\frac{B_{r} s^{r}+B_{r-1} s^{r-1}+\cdots+B_{1} s+B_{0}}{A_{r} s^{r}+A_{r-1} s^{r-1}+\cdots+A_{1} s+A_{0}}\right),
\end{aligned}
$$

where $B_{i}=b_{r-i}(r-i) !, A_{i}=a_{r-i}(r-i) !$, and $i=0,1,2, \ldots, r$.

The technical condition of the blade is then evaluated by calculating relative changes $\Delta A_{i}, \Delta B_{i}$ in model parameters, defined as follows: 


$$
\begin{aligned}
\Delta A_{i} & =\frac{A_{i}-\bar{A}_{i}}{\bar{A}_{i}}, \\
\Delta B_{i} & =\frac{B_{i}-\bar{B}_{i}}{\bar{B}_{i}},
\end{aligned}
$$

where $A_{i}, B_{i}$ are values of the $\varphi_{21}(\omega)$ model parameters evaluated at the moment $\vartheta_{1}$ and $\bar{A}_{i}, \bar{B}_{i}$ are mean values of these parameters evaluated during subsequent measurements within the period between $\vartheta_{0}$ (beginning of the monitoring process) and $\vartheta_{1}$ (the current moment at the monitoring process). Here, $t$ is the time in terms of Newton's definition (for diagnostic examinations) and $\vartheta$ is the time in terms of Bergson's definition (for diagnostic inference).

To simplify the evaluation of technical condition of a given blade, relative changes $\Delta A_{i}, \Delta B_{i}$ of the $\varphi_{21}(\omega)$ diagnostic model are classified into three damage threshold ranges and presented in a convenient graphical way, termed as a "damage map" [33]. The damage map of the blade is created as described below.

The three damage threshold ranges are defined with mean $\overline{\Delta A}_{i}, \overline{\Delta B}_{i}$ and standard deviation $\sigma_{A_{i}}, \sigma_{B_{i}}$ values of relative changes $\Delta A_{i}, \Delta B_{i}$, where

$$
\begin{aligned}
& \overline{\Delta A}_{i}=\frac{1}{K} \sum_{k}^{K} \Delta A_{i}(k), \\
& \overline{\Delta B}_{i}=\frac{1}{K} \sum_{k}^{K} \Delta B_{i}(k),
\end{aligned}
$$

and

$$
\begin{gathered}
\sigma_{A_{i}}=\sqrt{\frac{1}{K} \sum_{k}^{K}\left(\Delta A_{i}(k)-\overline{\Delta A}_{i}\right)^{2}}, \\
\sigma_{B_{i}}=\sqrt{\frac{1}{K} \sum_{k}^{K}\left(\Delta B_{i}(k)-\overline{\Delta B}_{i}\right)^{2}} .
\end{gathered}
$$

Here, $\Delta A_{i}(k)$ and $\Delta B_{i}(k)$ are relative changes in model parameters evaluated at time moment $\vartheta_{k}, \vartheta_{0} \leq \vartheta_{k} \leq \vartheta_{1}$, and $K$ is the number of those evaluations. For simplicity, $\Delta A_{i}, \Delta B_{i}$ are further denoted as $\Delta p, \overline{\Delta A}_{i}, \overline{\Delta B}_{i}$ as $\mu$, and $\sigma_{A_{i}}, \sigma_{B_{i}}$ as $\sigma$.

Next, three damage threshold ranges are assumed and the damage map is created. The map has a form of a color table of $K$ rows and $2 r$ columns, where each row contains color indications of whether at a given time moment $\vartheta_{k}$ the value of a given relative change $\Delta p$ falls within one of the three damage ranges. The colors in subsequent cells in a given row are assigned as follows:

(1) If at a given time moment $\vartheta_{k}$, relative changes $\Delta p$ are within the first damage threshold range, i.e., if

$$
(\mu-\sigma) \leq \Delta p \leq(\mu+\sigma)
$$

then the indication of $\Delta p$ in the $k$ th row, $i$ th column becomes green.

(2) If relative changes $\Delta p$ are within the second damage threshold range:

$$
(\mu-2 \sigma) \leq \Delta p \leq(\mu-\sigma) \text { or }(\mu+\sigma) \leq \Delta p \leq(\mu+2 \sigma),
$$

then the indication in the $k$ th row, $i$ th column becomes blue.

(3) If relative changes $\Delta p$ are within the third damage threshold range:

$$
(\mu-3 \sigma) \leq \Delta p \leq(\mu-2 \sigma) \text { or }(\mu+2 \sigma) \leq \Delta p \leq(\mu+2 \sigma),
$$
red.

then the indication in the $k$ th row, ith column becomes

(4) If relative changes $\Delta p$ are beyond any of the three damage threshold ranges, then the indication in the $k$ th row, ith column becomes black.

From the above procedure, it is clear that predominant black and red fields indicate a serious blade failure, predominant dark blue fields indicate excessive wear of the blade, and predominant green fields indicate slight or no wear of the blade.

The algorithm of the proposed blade evaluation technique is presented in Figure 2.

\section{Experimental Test Stand}

The proposed method has been verified experimentally at a turbine engine test stand at Air Force Institute of Technology, Warsaw (Figure 3). The tests have been performed for first stage blades of an axial compressor of SO-3 turbine engine mounted in TS-11 "Iskra" aircrafts [39-41].

The contactless inductive sensor designed at Air Force Institute of Technology [26, 42] and mounted at the engine chassis has been used to measure blades displacements. Its principle of operation is based on electrodynamical interaction and therefore it may be referred as a passive eddycurrent sensor. The sensor measures the signal of momentary location of the compressor blade tips during engine operation in time when blade approaches and recedes from the sensor (tip-timing method).

\section{Results}

An example blade displacement signal obtained with a sampling frequency of $500 \mathrm{kHz}$ is presented in Figure 4 . Three ranges of engine rotating speed may be seen: 1st at $6900 \mathrm{rpm}, 2 \mathrm{nd}$ at $12600 \mathrm{rpm}$, and $3 \mathrm{rd}$ at $15300 \mathrm{rpm}$.

The analysis has been conducted for the rotating speed of $12600 \mathrm{rpm}$, i.e., for the second range in Figure 4. In that range, 364000 peaks of blade tips displacement have been recorded. Since the compressor blade annulus has 28 blades, each blade moves 13000 times beneath the sensor. The 


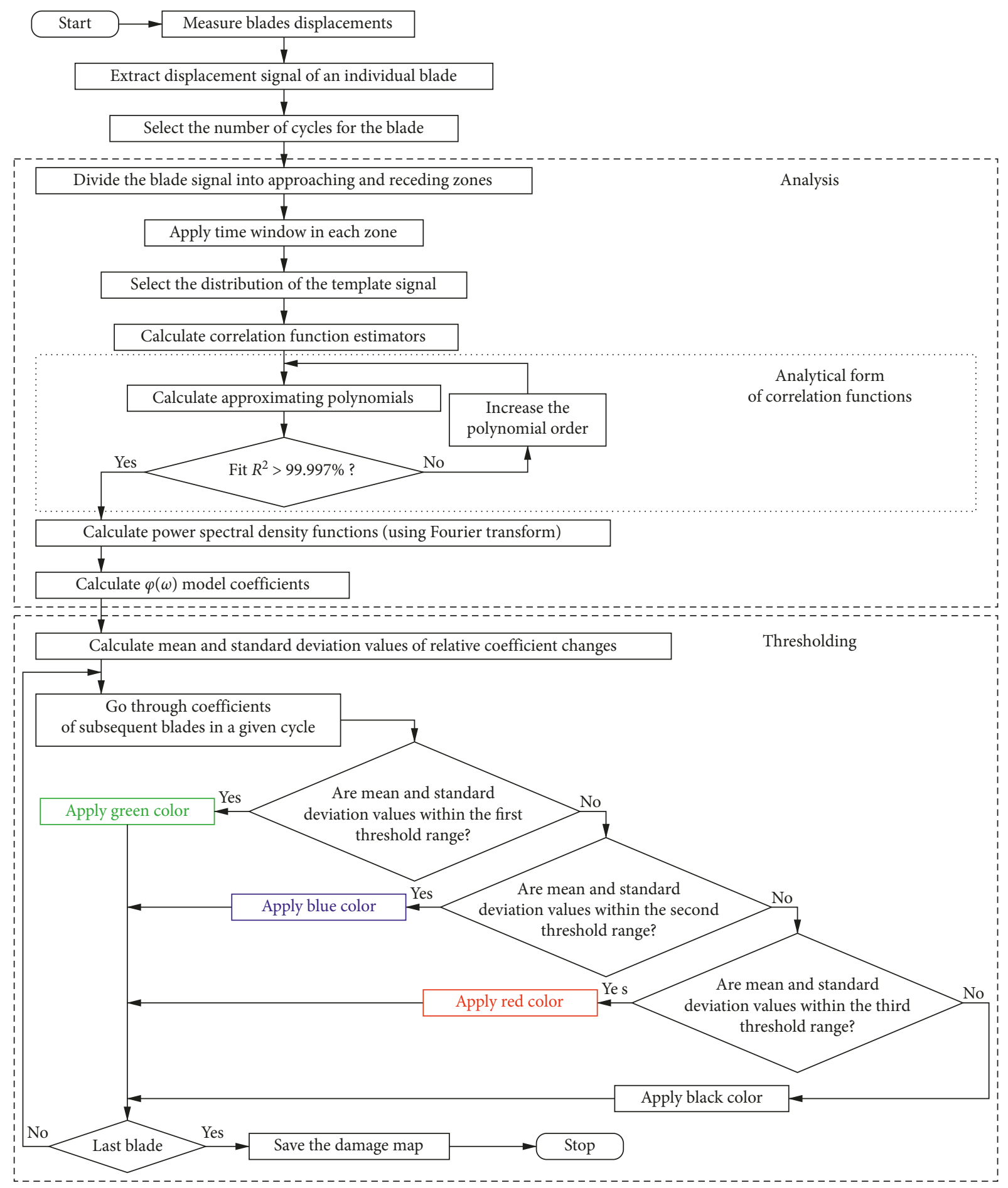

FIgUre 2: Algorithm of the blade evaluation technique.

displacement of each of 28 blades beneath the sensor is termed as "one cycle." The details of selected peaks in blade Nos. 3 and 8 displacement cycles measured at $12600 \mathrm{rpm}$ (2nd range of the rotating speed) are shown in Figure 5. As can be seen, the quality of the displacement signals obtained with the special inductive sensor described above is very good.
For blade analysis, 36 out of 13000 cycles are selected to form a statistical sample. To quickly form a sample, the selection is made in one of three ways:

(i) Logarithmic selection: 36 cycles are selected in such a way that each one from 1 to 9 , each 10th from 19 to 90, each 100th from 100 to 900, and each 1000th from 1000 to 9000 form the sample 

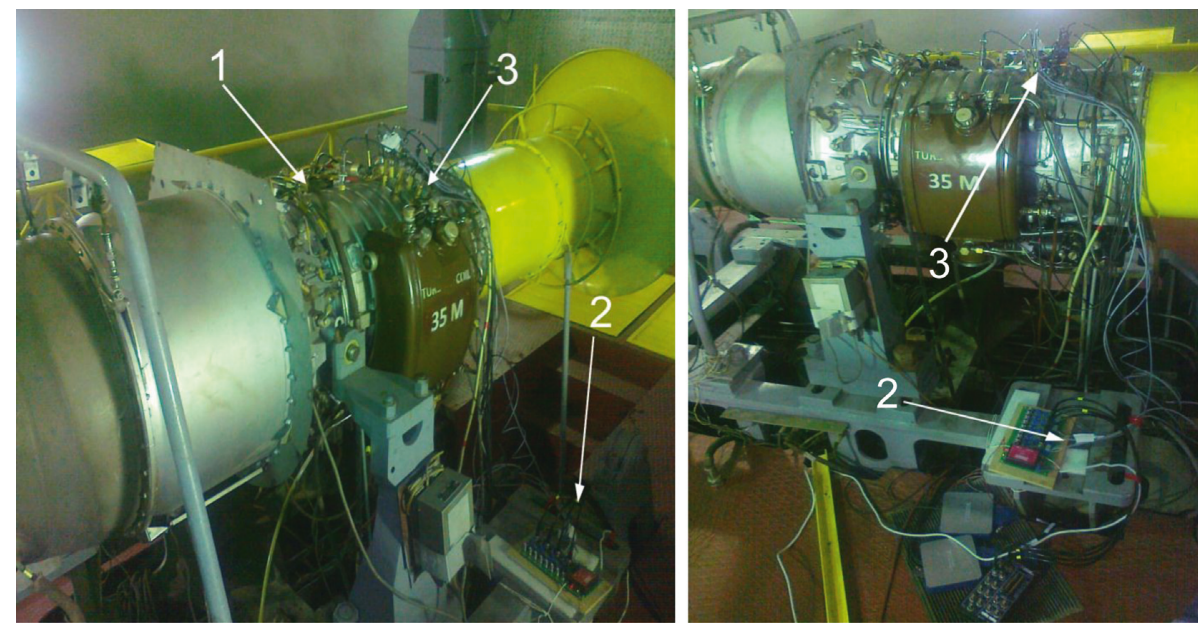

Figure 3: Experimental test stand: (1) turbine engine, (2) signal amplifier, and (3) contactless inductive sensor [39].

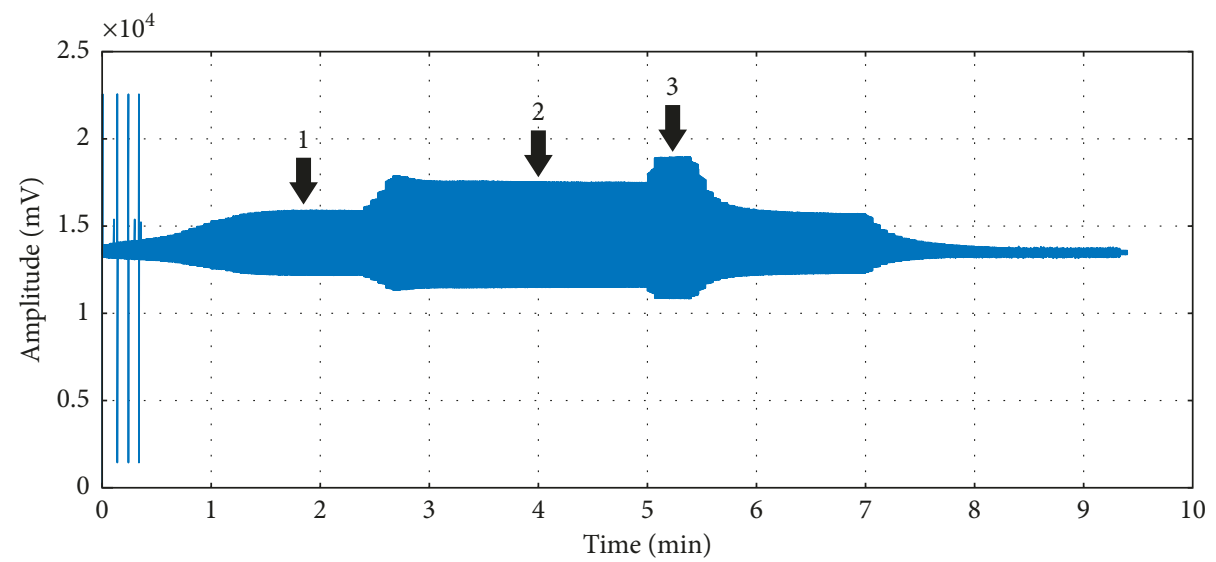

Figure 4: Blade tips displacement at three rotating speeds: (1) $6900 \mathrm{rpm}$, (2) $12600 \mathrm{rpm}$, and (3) $15300 \mathrm{rpm}$.

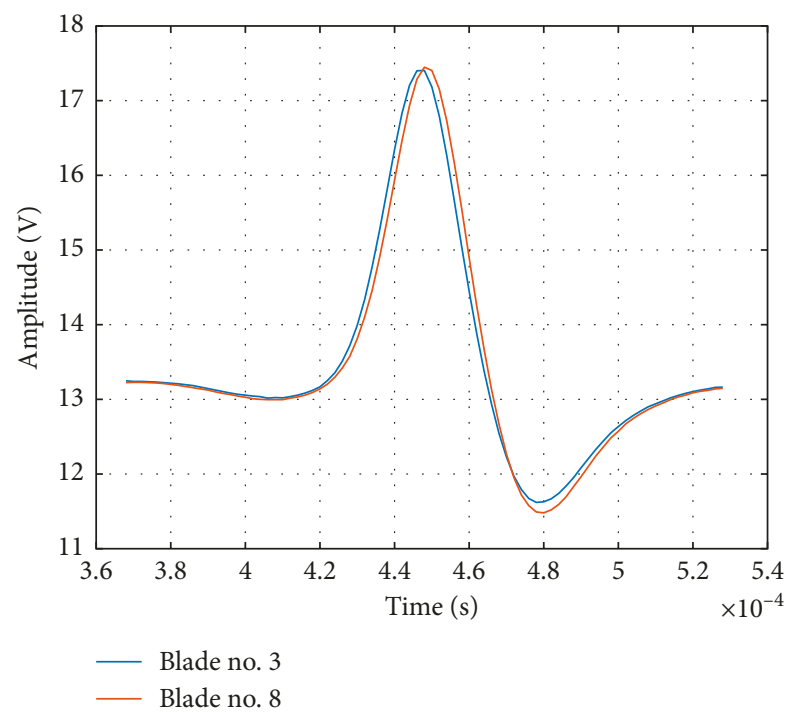

Figure 5: Selected peaks in blades displacement cycles at $12600 \mathrm{rpm}$. 


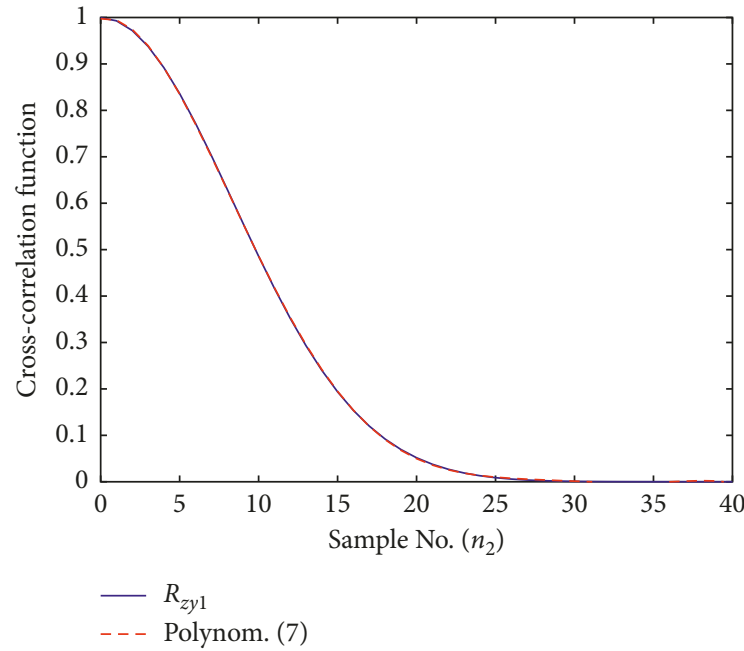

(a)

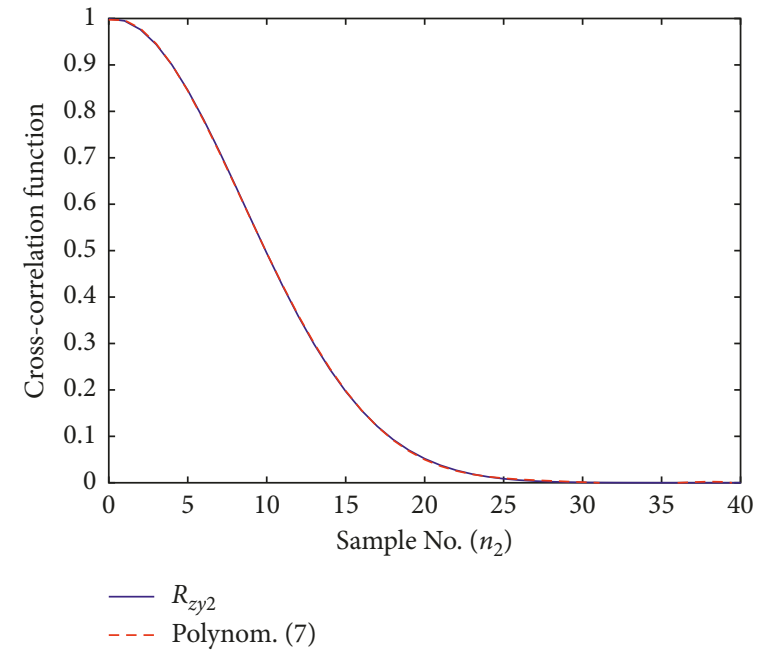

(b)

FIGURE 6: Cross-correlation functions $R_{z y 1}\left(n_{2}\right), R_{z y 2}\left(n_{2}\right)$ and their polynomial approximations $R_{z y 1}(\tau), R_{z y 2}(\tau)$ for blade No. 3 at (a) the approaching zone $\Delta t_{1}$ and (b) the receding zone $\Delta t_{2}$.

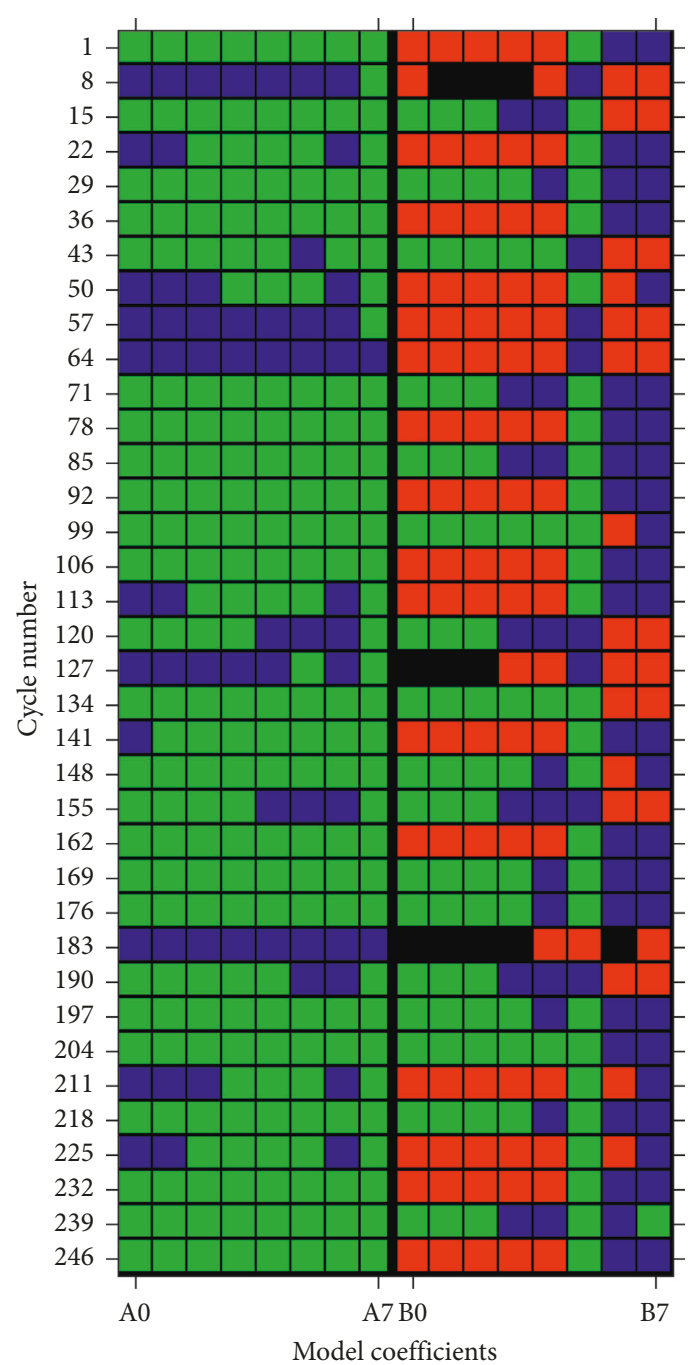

(a)

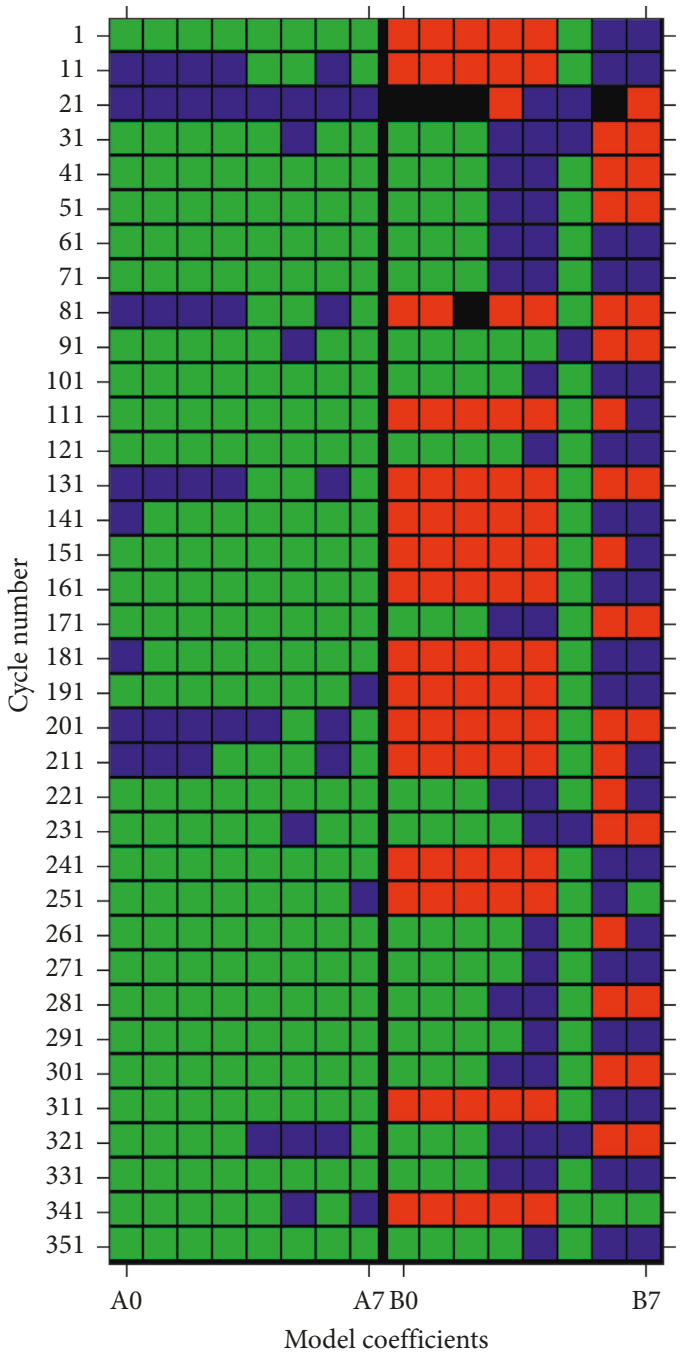

(b)

Figure 7: Damage maps of blade No. 3 for (a) each 7th cycle and (b) each 10th cycle. 


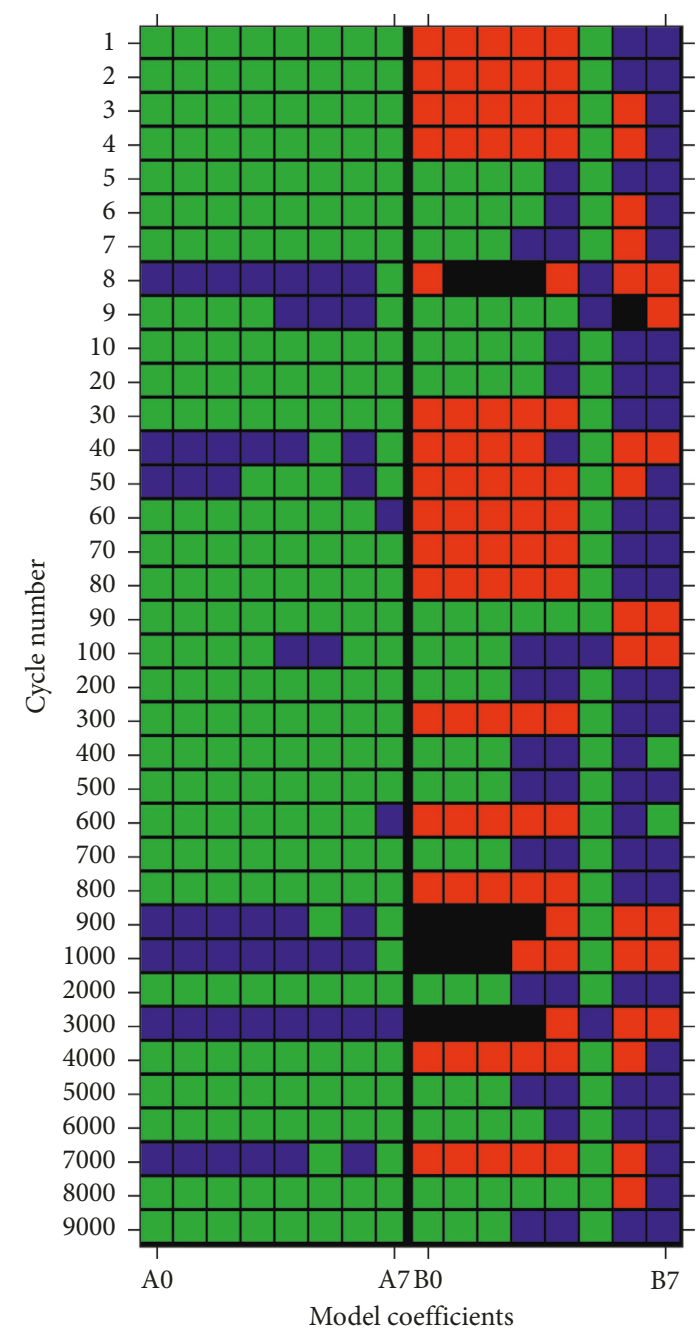

(a)

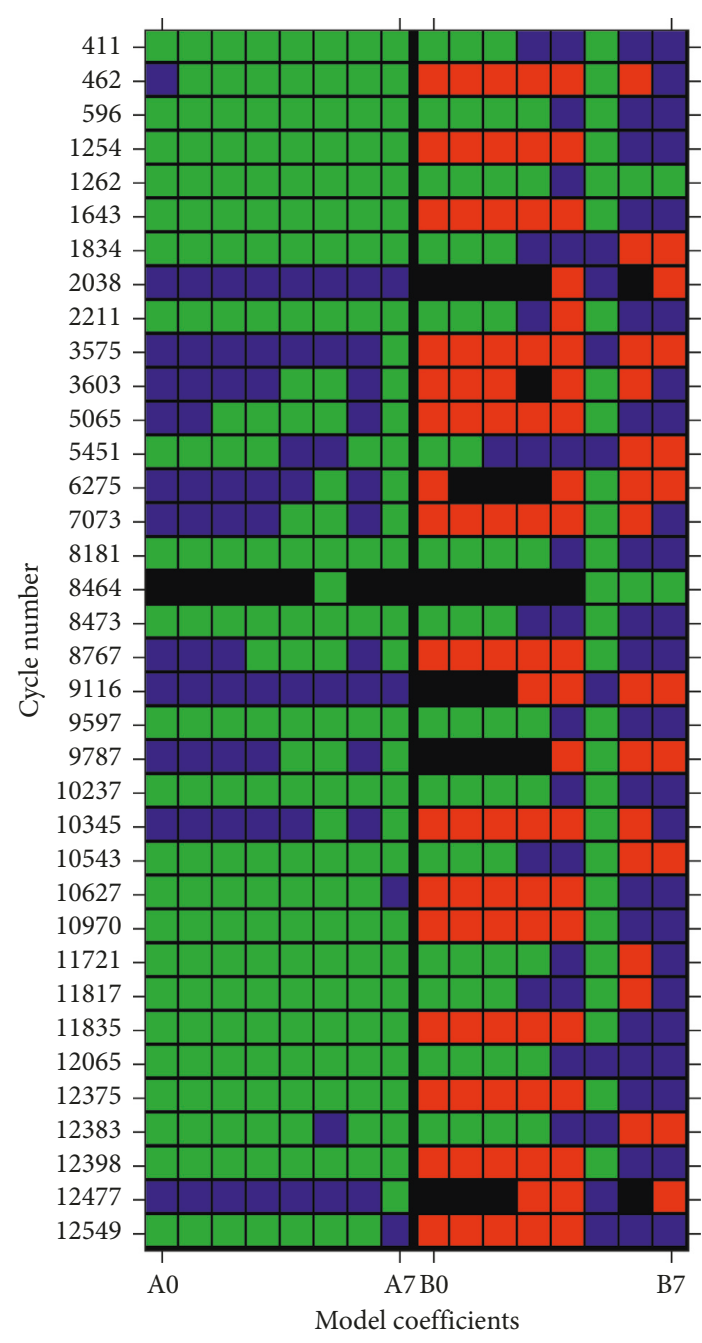

(b)

Figure 8: Damage maps of blade No. 3 for (a) logarithmic and (b) random selection of cycles.

(ii) Selection of each $n$th cycle: the first cycle, the cycle selection step, and the number of cycles are indicated

\section{(iii) Random selection}

It is expected that damage maps obtained for each selection give the same image of blade technical condition (which will confirm the efficiency of the method). The damage maps are presented for two blades out of 28 in the annulus. The blades have been chosen in such a way that the first is in the best (blade No. 8), and the other is in the worst (blade No. 3) technical condition.

After several initial calculations, the order of the approximating polynomials of cross-correlation functions has been selected as $r=7$, resulting in the coefficient of determination $R^{2}=99.95 \%$. Lower polynomial orders lead to lower determination coefficient values (i.e., to less accurate approximations). Higher orders do not improve approximation's accuracy noticeably (as $R^{2}$ is already close to 1 ), yet considerably increase the calculation time. The amplitude parameter of environmental signal has been assumed as $T=15$.

Example cross-correlation functions $R_{z y 1}(m), R_{z y 2}(m)$ and their 7 th order polynomial approximations $R_{z y 1}(\tau)$, $R_{z y 2}(\tau)$ at the two observation zones of the selected peak of blade No. 3 displacement signal (shown in Figure 5) are presented in Figure 6.

Thus, the technical condition of each blade is represented by 16 coefficients: 8 in the approaching and 8 in the receding zones. These zones are clearly visible as left and right parts of damage maps in Figures 7-10. The samples have been selected using the $n$th selection (Figures 7 (a) and 9(a) for each 7th cycle and Figures 7(b) and 9(b) for each 10th cycle), the logarithmic selection (Figures 8(a) and 10(a)), and the random selection (Figures $8(\mathrm{~b})$ and 10(b)).

As can be seen, the technical condition of each blade is clearly and explicitly indicated in the obtained damage maps. Predominant red and black fields in Figures 7 and 8 are 


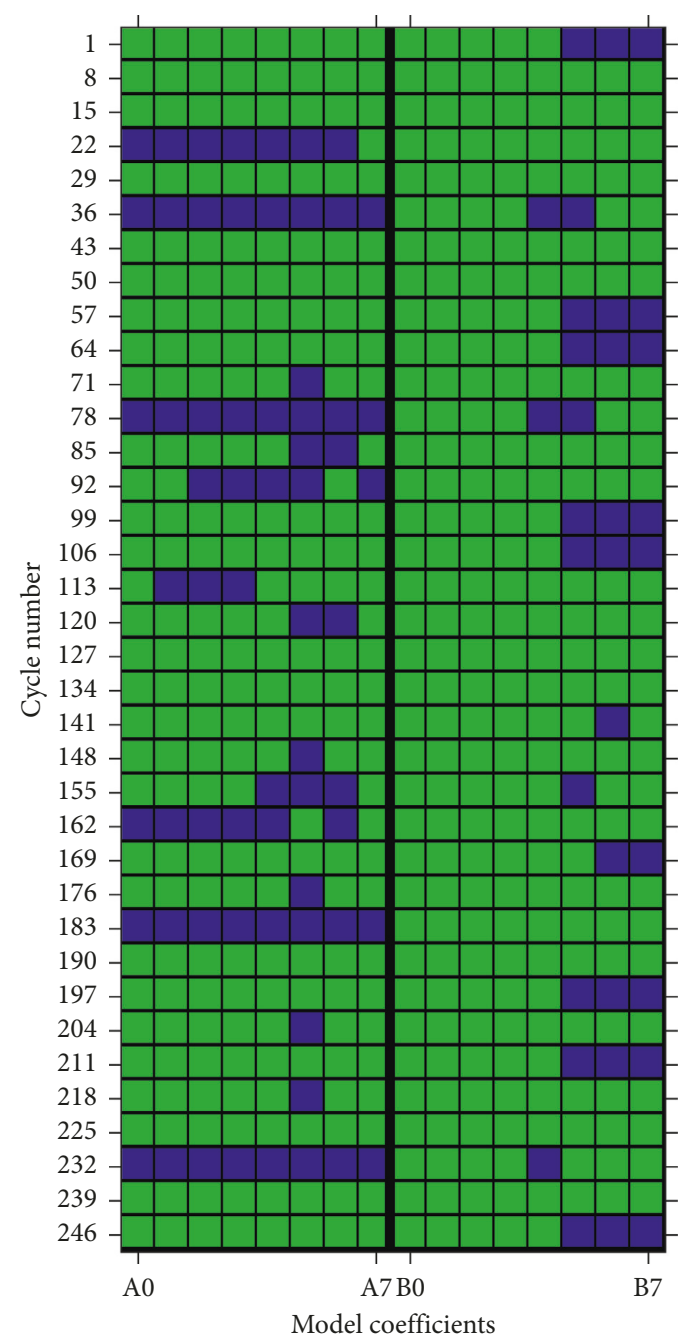

(a)

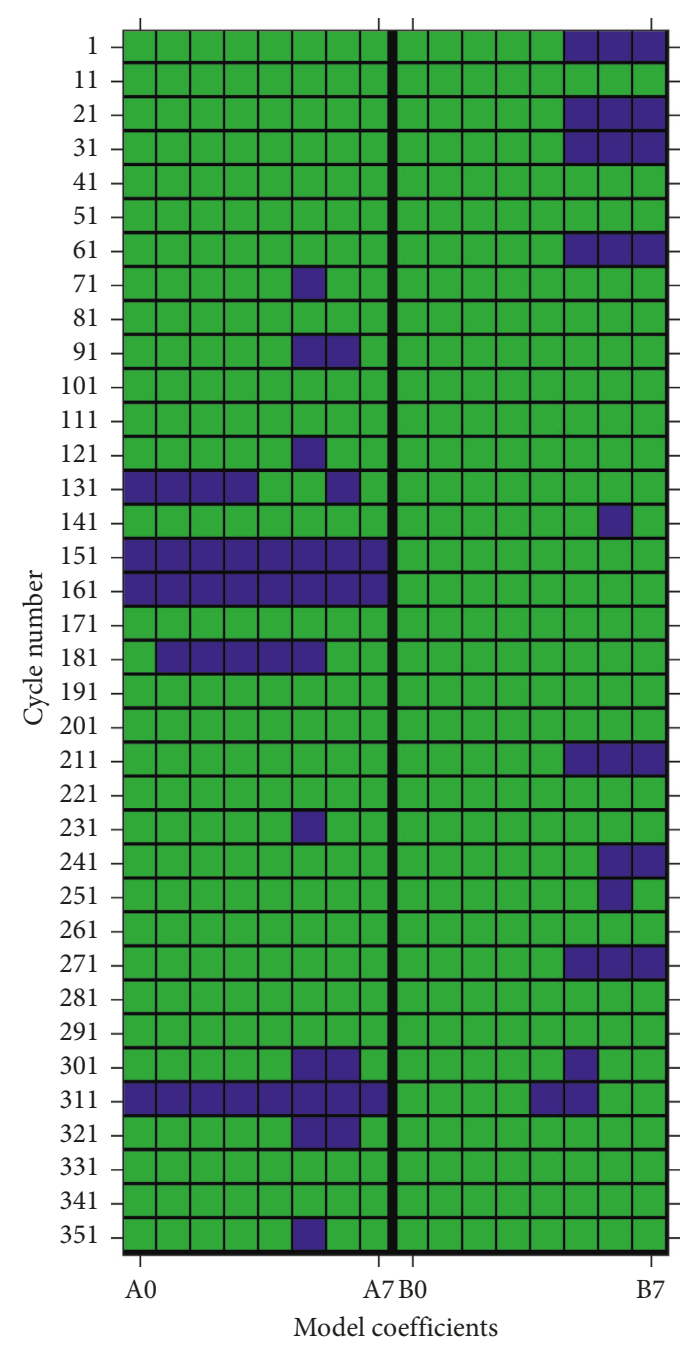

(b)

Figure 9: Damage maps of blade No. 8 for (a) each 7th cycle and (b) each 10th cycle.

characteristics for the seriously damaged blade No. 3. For blade No. 8, the obtained damage maps (Figures 9 and 10) are almost green, confirming its relatively good technical condition. These results are independent of the selection type: the samples of $n$ th, logarithmic, or random selection types give similar, clear indications of the technical condition of the blade. As damage thresholds are based on $\mu \pm \sigma$, $\mu \pm 2 \sigma$, and $\mu \pm 3 \sigma$ statistics, small deviations between the colors of a given damage map are possible. Therefore, it is important to analyze the map globally, for a reasonable number of rotation cycles (rows of the map).

Using damage maps, the technical condition of all 28 blades can be presented in a compact, graphical form as shown in Figure 11. This form is called the damage portrait of the blades in the annulus. At horizontal axis, all 28 blades are numbered in an ascending order. At vertical axis, the number of fields of a given color (green, blue, red, and black) at the related damage map is marked with a colored circle. As can be seen for blade No. 3, the number of red fields is high (above 100) while the number of green fields is low (below 350). This suggests a possible serious blade wear (or damage). For blade No. 8, the number of black, red, and blue fields is low (below 100), while the number of green fields is high (almost 500). This indicates that the technical condition of this blade is good.

The presented results reflect the state of each blade only in terms of its "better" or "worse" technical condition, as no real damage is introduced to the test stand. Experimental tests of the blade with a serious damage are difficult or even impossible to conduct as may quickly lead to a dangerous failure of the machine.

\section{Summary}

The proposed method of blades technical condition evaluation is based on a diagnostic model having the form of the phase shift between the cross-correlation functions of the measured vibration signal and an additional template signal 


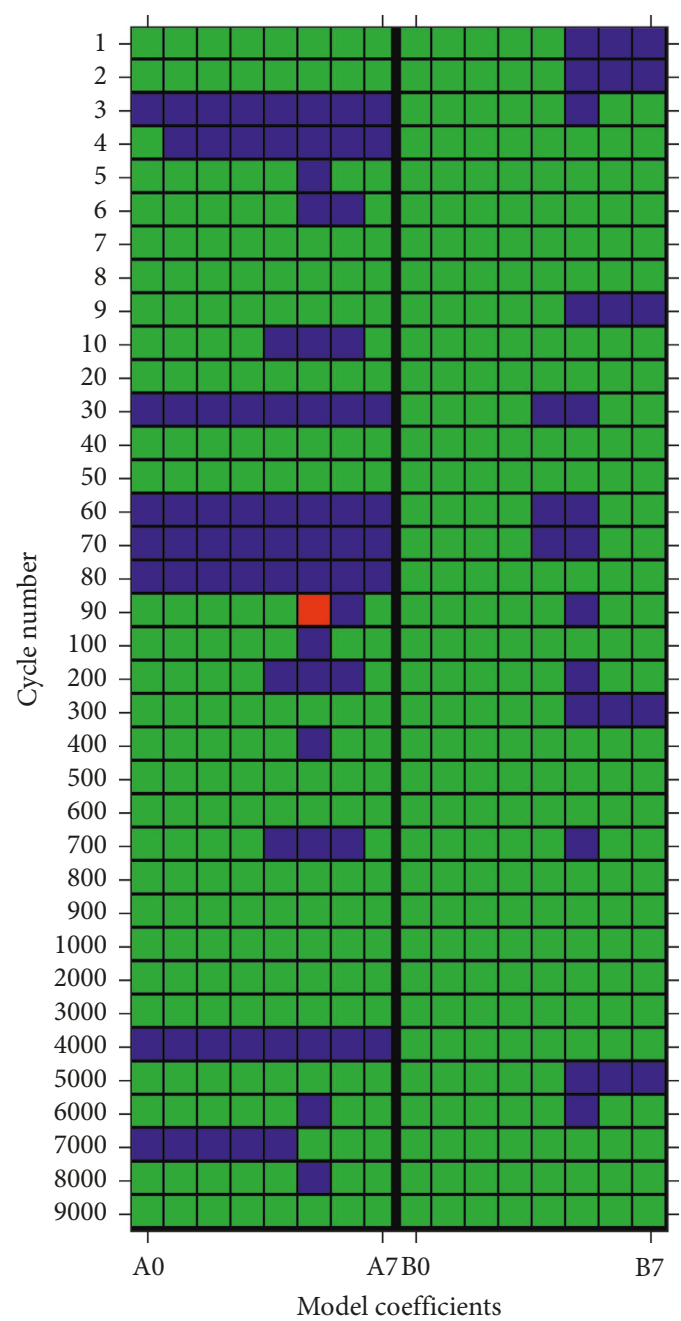

(a)

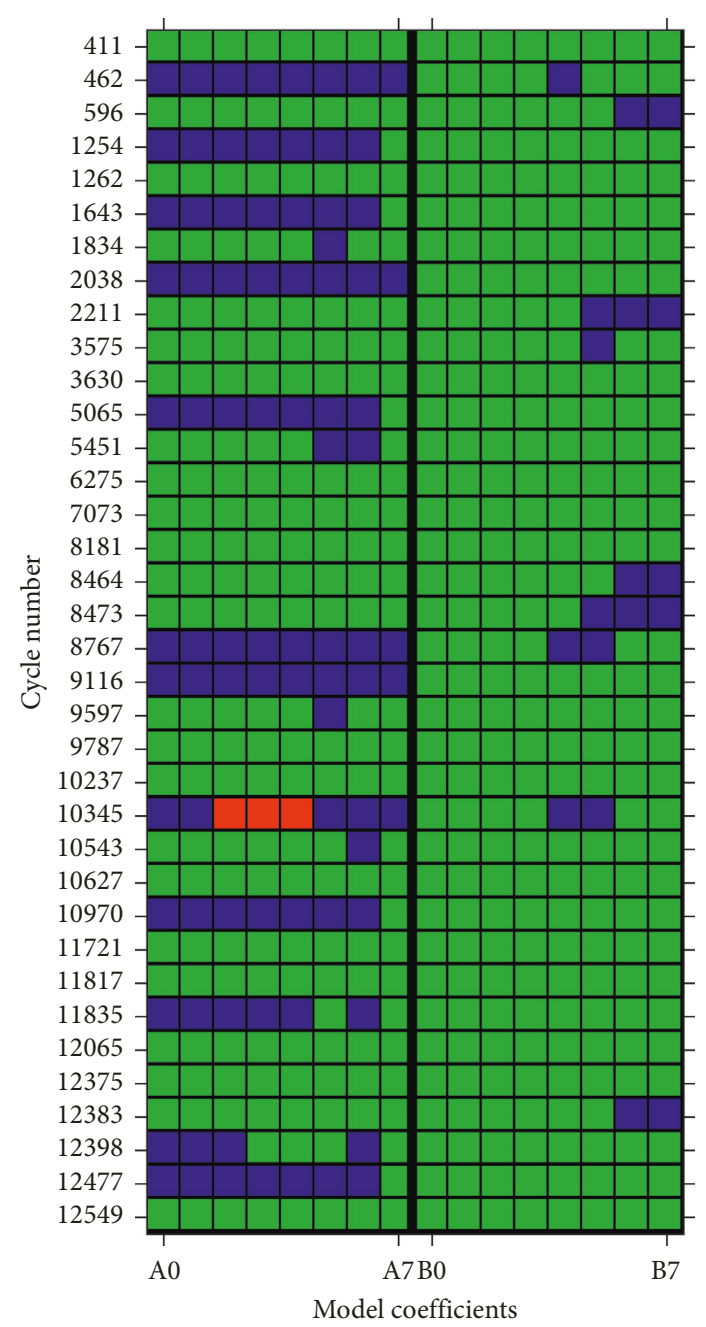

(b)

FIgURE 10: Damage maps of blade No. 8 for (a) logarithmic and (b) random selection of cycles.

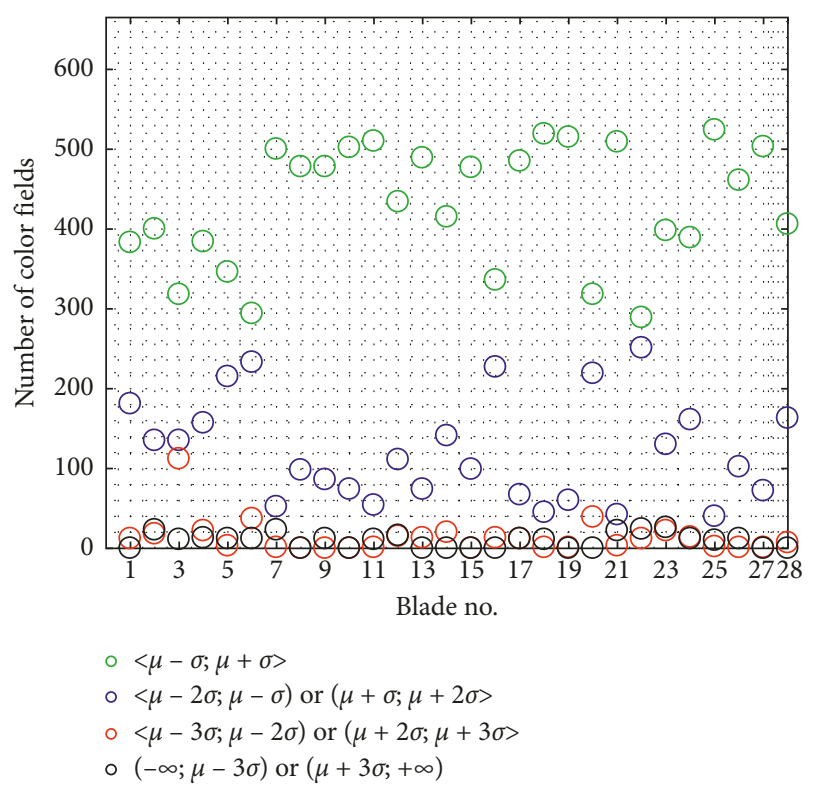

FIgURE 11: Damage portrait of all 28 blades in the annulus. calculated in two subsequent observation zones: when the blade approaches the sensor and when the blade recedes the sensor. If the time distance between these zones is short enough, then environmental signals affecting the operational signal in both zones can be assumed as identical. By taking this assumption, the direct measurement of the environmental signal is not required, yet its influence is indirectly eliminated by a special organization of the diagnostic process. This way the reliability of the machine technical condition indications is improved, which has been demonstrated by experimental results obtained at the turbine engine test stand. The technical condition of each blade has been projected into damage maps representing the relative changes in the parameters of the diagnostic model. Furthermore, the technical condition of all blades in the annulus has been presented in a compact, simple to interpret damage portrait. Such graphical representations are communicative and can be used to quickly interpret the technical condition of the machine.

The proposed method can be used to assess the technical condition of advanced rotating machines, such as turbine 
engines. It can be applied online with no need to disassembly the engine and gives early damage indications to plan overhauls accurately.

\section{Data Availability}

The data used to support the findings of this study are available from the corresponding author upon request.

\section{Conflicts of Interest}

The authors declare that they have no conflicts of interest.

\section{Acknowledgments}

This paper was supported by the Bialystok University of Technology under research project nos. W/WM/9/2013 and S/WM/1/2016.

\section{References}

[1] M. Lackner, "Vibration and crack detection in gas turbine engine compressor blades using eddy current sensors," M.Sc. thesis, Massachusetts Institute of Technology, Cambridge, MA, USA, 2003.

[2] Z. Bai, S. Chen, L. Jia, and Z. Zeng, "Phased array ultrasonic signal compressive detection in low-pressure turbine disc," NDT\&E International, vol. 89, pp. 1-13, 2017.

[3] S. Yang, B. Yoon, and Y. Kim, "Using phased array ultrasonic technique for the inspection of straddle mount-type lowpressure turbine disc," NDT\&E International, vol. 42, no. 2, pp. 128-132, 2009.

[4] J. Blachnio, "Capabilities to assess health/maintenance status of gas turbine blades with non-destructive methods," Polish Maritime Research, vol. 21, no. 4, pp. 41-47, 2014.

[5] C. Meola, G. M. Carlomagno, M. di Foggia, and O. Natale, "Infrared thermography to detect residual ceramic in gas turbine blades," Applied Physics A, vol. 91, pp. 685-691, 2008.

[6] T. Saravanan, S. Bagavathiappan, J. Phillip, T. Jayakumar, and B. Raj, "Enhanced sensitivity detection of defects in gas turbine blades of aero-engine and hairpin tubes of heavy water plant using microfocal radiography," Radiography, vol. 50, no. 10, pp. 560-563, 2008.

[7] J. M. Barragan, Engine Vibration Monitoring and Diagnosis Based on On-Board Captured Data, DTIC Document ADP014124, MTU Aero Engines Gmbh Munich, München, Germany, 2013.

[8] H. R. Simmons, "Non-intrusive detection of turbine blade resonance," in Proceedings of 3 rd EPRI Conference on Incipient Failure Detection in Power Plants, Philadelphia, PA, USA, May 1987.

[9] P. Parge, B. Trevillion, and P. Carle, "Non-intrusive vibration monitoring for turbine blade reliability," in Proceedings of the 2nd International Machinery Monitoring and Diagnostic Conference, Los Angeles, CA, USA, October 1990.

[10] F. Al-Badour, M. Sunar, and L. Cheded, "Vibration analysis of rotating machinery using time-frequency analysis and wavelet techniques," Mechanical Systems and Signal Processing, vol. 25, no. 6, pp. 2083-2101, 2011.

[11] A. M. Abdelrhman, L. M. Hee, M. S. Leong, and S. Al-Obaidi, "Condition monitoring of blade in turbomachinery: a review," Advances in Mechanical Engineering, vol. 6, no. 210717, pp. 1-10, 2014.
[12] M. Satyam, V. S. Rao, and C. G. Devy, "Cepstrum analysis-an advanced technique in vibration analysis of defects in rotating machinery," Defence Science Journal, vol. 44, pp. 53-60, 1994.

[13] R. Randall and N. Sawalhi, "Use of cepstrum to remove selected discrete frequency components from a time signal" Rotating Machinery," Structural Health Monitoring, Shock and Vibration, vol. 5, pp. 451-461, 2011.

[14] C. Chang and L. Chen, "Damage detection of cracked thick rotating blades by a spatial wavelet based approach," Applied Acoustics, vol. 65, no. 11, pp. 1095-1111, 2004.

[15] N. Aretakis and K. Mathioudakis, "Wavelet analysis for gas turbine fault diagnostics," ASME Journal of Engineering for Gas Turbines and Power, vol. 119, no. 4, pp. 870-875, 1997.

[16] M. H. Lim and M. S. Leong, "Detection of early faults in rotating machinery based on wavelet analysis," Advances in Mechanical Engineering, vol. 5, no. 625863, pp. 1-8, 2013.

[17] H. Jiang, C. Li, and H. Li, "An improved EEMD with multiwavelet packet for rotating machinery multi-fault diagnosis," Mechanical Systems and Signal Processing, vol. 36, no. 2, pp. 225-239, 2013.

[18] G. Battiato, C. M. Firrone, and T. M. Berruti, "Forced response of rotating bladed disks: blade tip-timing measurements," Mechanical Systems and Signal Processing, vol. 85, pp. 912-926, 2017.

[19] H. R. Simmons, D. L. Michalsky, K. E. Brewer, and A. J. Smalley, "Measuring rotor and blade dynamics using an optical blade tip sensor," in Proceedings of the 35th ASME International Gas Turbine and Aeroengine Congress and Exposition, Brussels, Belgium, June 1990.

[20] S. Madhavan, R. Jain, C. Sujatha, and A. S. Sekhar, "Vibration based damage detection of rotor blades in a gas turbine engine," Engineering Failure Analysis, vol. 46, pp. 26-39, 2014.

[21] A. G. Sheard, "Blade by blade tip clearance measurement," International Journal of Rotating Machinery, vol. 2011, Article ID 516128, 13 pages, 2011.

[22] A. Steiner, "Techniques for blade tip clearance measurements with capacitive probes," Measurement Science and Technology, vol. 11, no. 7, pp. 865-869, 2000.

[23] Z. Chen, Y. Yang, Y. Xie, B. Guo, and Z. Hu, "Non-contact crack detection of high-speed blades based on principal component analysis and Euclidian angles using optical-fiber sensors," Sensors and Actuators A: Physical, vol. 201, pp. 66-72, 2013.

[24] H. Guo, F. Duan, and J. Zhang, "Blade resonance parameter identification based on tip-timing method without the onceper revolution sensor," Mechanical Systems and Signal Processing, vol. 66-67, pp. 625-639, 2016.

[25] M. Neumann, F. Dreier, P. Gunther et al., "A laser-optical sensor system for blade vibration detection of high-speed compressors," Mechanical Systems and Signal Processing, vol. 64-65, pp. 337-346, 2015.

[26] E. Rokicki, J. Spychala, R. Szczepanik, and P. Majewski, Induction Sensor to Measure Vibrations of a Turbo-Machine Rotor Blade, US Patent No. 8,240,212 B2, U.S. Patent and Trademark Office, Washington, DC, USA, 2012.

[27] R. Rzadkowski, E. Rokicki, L. Piechowski, and R. Szczepanik, "Analysis of middle bearing failure in rotor jet engine using tip-timing and tip-clearance techniques," Mechanical Systems and Signal Processing, vol. 76-77, pp. 213-227, 2016.

[28] M. Annerfeldt, S. Shukin, M. Bjorkman, A. Karlsson, A. Jonsson, and E. Svistunova, "GTX100 turbine section measurement using a temperature sensitive crystal technique. A comparison with 3D thermal and aerodynamic analysis," in Proceedings of the 12th Annual Power-Gen Europe Conference 
and Exhibition, Power-Gen Europe, Barcelona, Spain, May 2004.

[29] K. M. Kim, J. S. Park, D. H. Lee, T. W. Lee, and H. H. Cho, "Analysis of conjugated heat transfer, stress and failure in a gas turbine blade with circular cooling passages," Engineering Failure Analysis, vol. 18, no. 4, pp. 1212-1227, 2011.

[30] K. Mathioudakis, A. Papathanasiou, E. Loukis, and K. Papailiou, "Fast response wall pressure measurement as a means of gas turbine blade fault identification," ASME Journal of Engineering for Gas Turbines and Power, vol. 113, no. 2, pp. 269-275, 1991.

[31] S. Al-Obaidi, M. S. Leong, R. Hamzah, and A. M. Abdelrhman, "A review of acoustic emission technique for machinery condition monitoring: defects detection and diagnostic," Applied Mechanics and Materials, vol. 229-231, pp. 1476-1480, 2012.

[32] O. Freund, M. Montgomery, M. Mittelbach, and J. R. Seume, "Non-contact test set-up for aeroelasticity in a rotating turbomachine combining a novel acoustic excitation system with tip-timing," Measurement Science and Technology, vol. 25, no. 3, article 035008, 2014.

[33] R. Gradzki, "Influence of diagnostic signal sampling frequency on rotor blade technical condition images determined from phase shift difference," Journal of KONES Powertrain and Transport, vol. 19, no. 2, pp. 181-189, 2012.

[34] R. Gradzki, "The influence of diagnostic signal measurement period on blades technical condition images determined from phase shift difference," Solid State Phenomena, vol. 199, pp. 67-72, 2013.

[35] P. Lindstedt and R. Gradzki, "Parametrical models of working rotor machine blade diagnostics with its unmeasurable environment elimination," Acta Mechanica et Automatica, vol. 4, no. 4, pp. 56-63, 2010.

[36] P. Lindstedt and R. Gradzki, "Premises and the example of parametric method of evaluation of technical condition of the turbomachine blade with elimination of its immeasurable environment," Journal of KONBiN, vol. 1, no. 17, pp. 179-194, 2011.

[37] R. F. Hoskins, Delta Functions. Introduction to Generalised Functions, 2nd edition, Woodhead Publishing, Cambridge, UK, 2009.

[38] C. Chatfield, The Analysis of Time Series-An Introduction, Chapman and Hall, London, UK, 2003.

[39] K. Golak, "Validity check of the assessment of a jet turbine engine regulation in flight using a computer simulation," Solid State Phenomena, vol. 199, pp. 123-128, 2013.

[40] K. Golak, P. Lindstedt, and R. Gradzki, "Studies of the jet engine control quality based on its response to the disturbance inflicted on the object, designated from its response to the set point inflicted to the controller," in Proceedings of the European Safety and Reliability Conference: Methodology and Applications 2014 (ESREL'14), pp. 137-140, Wroclaw, Poland, September 2014.

[41] K. Kawalec, W. Balicki, R. Chachurski et al., Aircraft Turbine Engines-Structures, Maintenance, Diagnostics, Institute of Aviation, Warsaw, Poland, 2012, in Polish.

[42] R. Przysowa and E. Rokicki, "Inductive sensors for blade tiptiming in gas turbines," Journal of KONBiN, vol. 4, no. 36, pp. 147-164, 2015. 


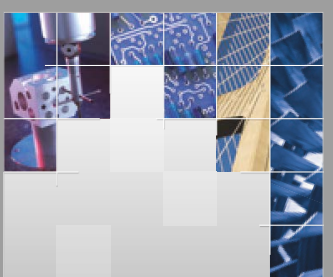

\section{Enfincering}
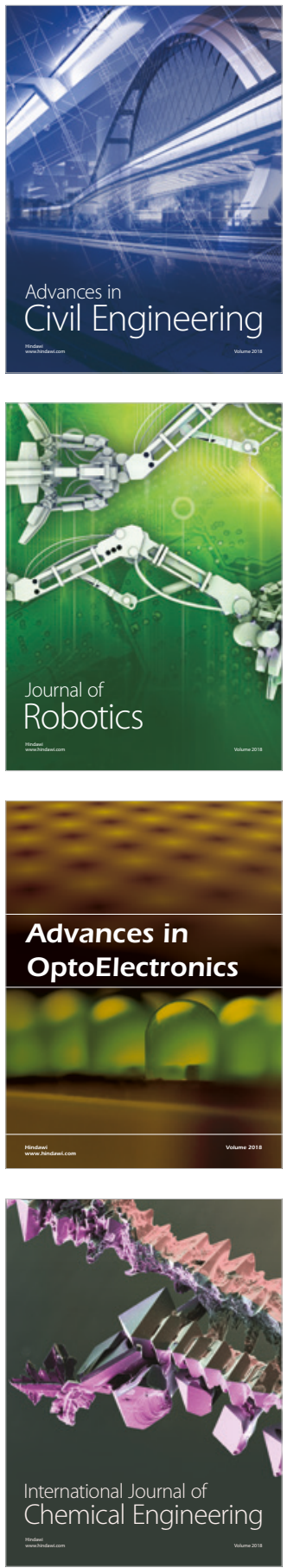

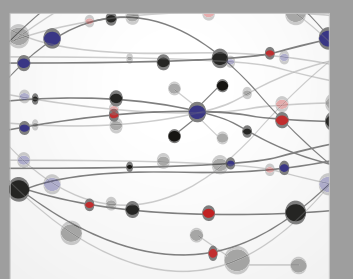

\section{Rotating \\ Machinery}

The Scientific World Journal

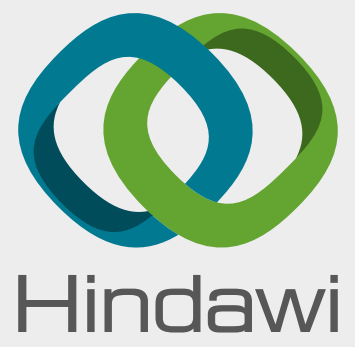

Submit your manuscripts at

www.hindawi.com
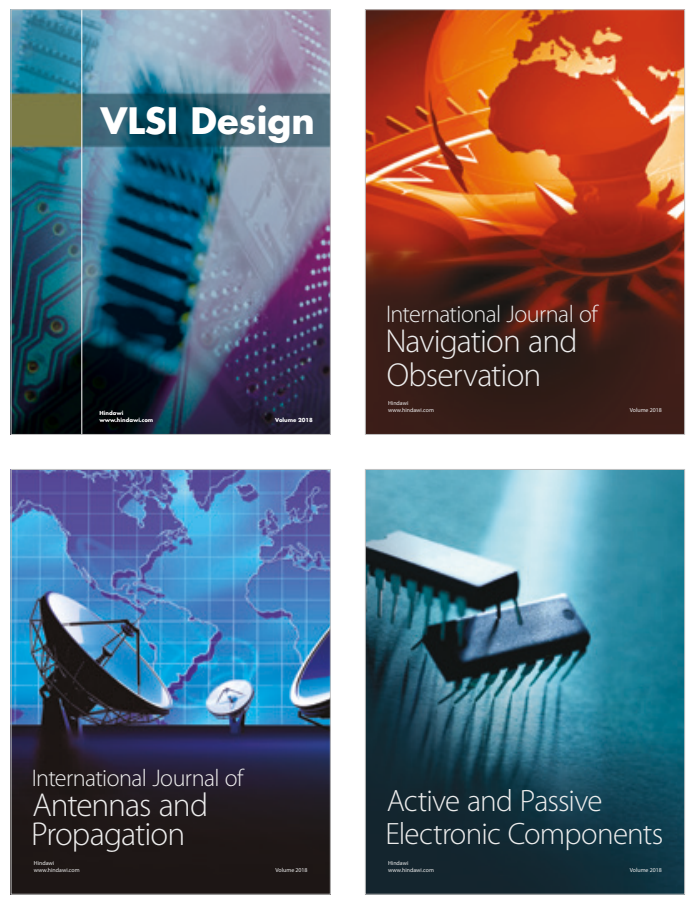
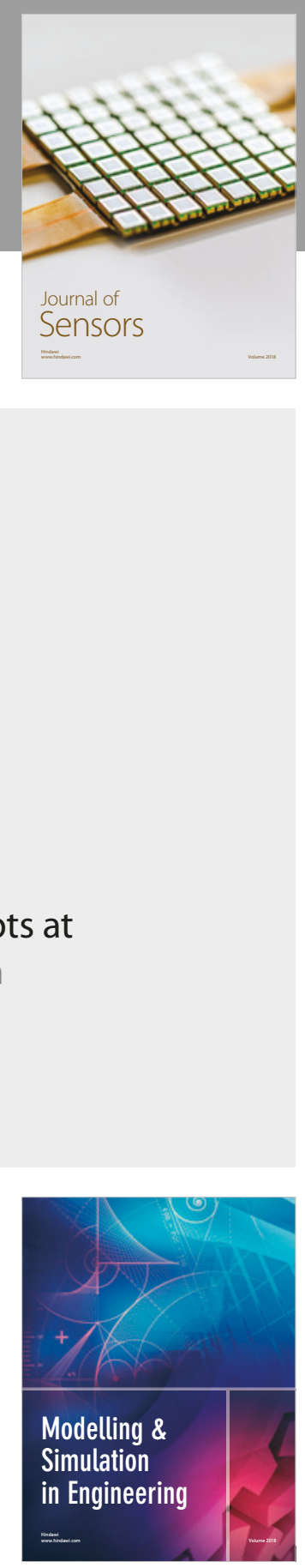

\section{Advances \\ Multimedia}
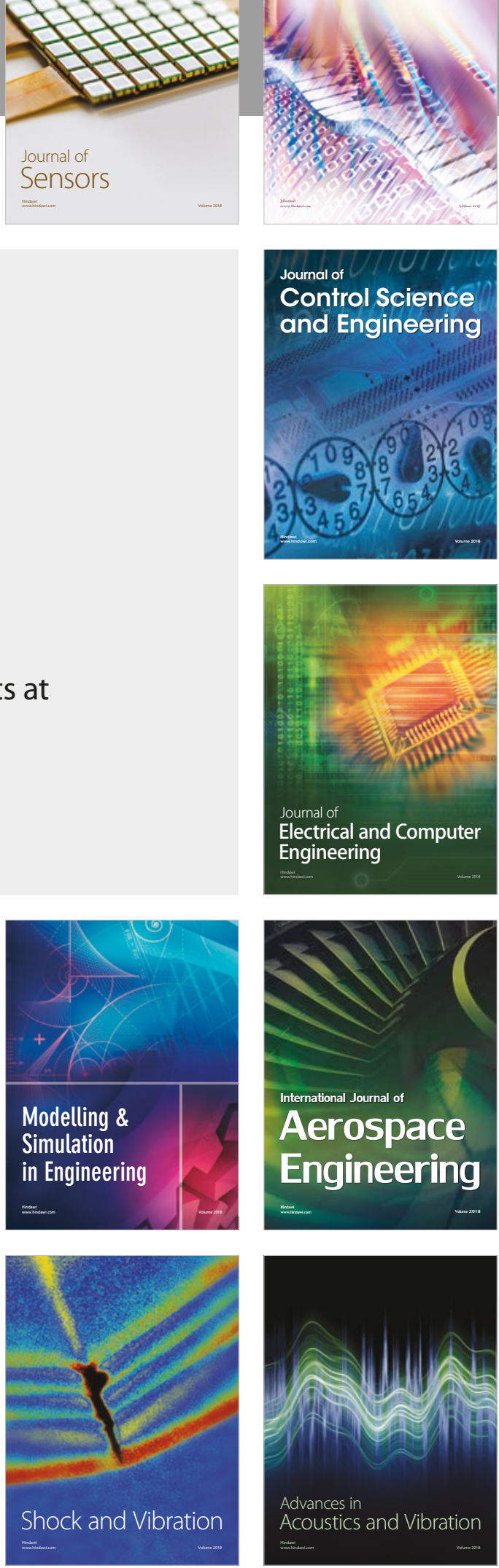